\title{
Article \\ Impact of Treating Asymptomatic Bacteriuria in Kidney Transplant Recipients: A Prospective Cohort Study
}

\author{
Sara Fontserè ${ }^{1,+}{ }^{\oplus}$, Carmen Infante-Domínguez ${ }^{1, \dagger}$, Alejandro Suárez-Benjumea ${ }^{2}$, Marta Suñer-Poblet ${ }^{2}$, \\ Carmen González-Corvillo ${ }^{2}$, Guillermo Martín-Gutiérrez ${ }^{1}$, Gabriel Bernal ${ }^{1}$, Jerónimo Pachón 1,3(1), \\ María Eugenia Pachón-Ibáñez ${ }^{1,4, * \mathbb{C}}$ and Elisa Cordero ${ }^{1,3,4}$
}

1 Unit of Infectious Diseases, Microbiology, and Preventive Medicine, Virgen del Rocío University Hospital of Seville, 41013 Seville, Spain; sarafontsere@gmail.com (S.F.); carmeninfanted@gmail.com (C.I.-D.); guiller_mg86@hotmail.es (G.M.-G.); gabrielbernalblanco@gmail.com (G.B.); pachon@us.es (J.P.); elisacorderom@gmail.com (E.C.)

2 Urology and Nephrology Unit, Virgen del Rocío University Hospital, 41013 Seville, Spain; alejandro.suarez.sspa@juntadeandalucia.es (A.S.-B.); msuner@us.es (M.S.-P.); carmen.gonzalez.corvillo.sspa@juntadeandalucia.es (C.G.-C.)

3 Department of Medicine, University of Seville, 41009 Seville, Spain

4 Institute of Biomedicine of Seville (IBiS), Virgen del Rocío University Hospital/CSIC/University of Seville, 41013 Seville, Spain

check for updates

Citation: Fontserè, S.;

Infante-Domínguez, C.;

Suárez-Benjumea, A.; Suñer-Poblet, M.; González-Corvillo, C.;

Martín-Gutiérrez, G.; Bernal, G.;

Pachón, J.; Pachón-Ibáñez, M.E.;

Cordero, E. Impact of Treating

Asymptomatic Bacteriuria in Kidney Transplant Recipients: A Prospective Cohort Study. Antibiotics 2021, 10, 218. https://doi.org/10.3390/ antibiotics 10020218

Academic Editor: Gabriella Orlando

Received: 4 February 2021

Accepted: 19 February 2021

Published: 22 February 2021

Publisher's Note: MDPI stays neutral with regard to jurisdictional claims in published maps and institutional affiliations.
* Correspondence: mpachon-ibis@us.es

$\dagger \quad$ The authors contributed equally to this work.

\begin{abstract}
This study aims to define the epidemiologic, clinical, and microbiological features of asymptomatic bacteriuria (AB) and cystitis in kidney transplantation recipients (KTRs), and to determine the impact of antimicrobial therapy of $\mathrm{AB}$ and the risk factors of cystitis. We conducted a prospective observational study of AB and cystitis in KTRs from January to June 2017. One-hundred ninety seven KTRs were included: 175 (88.8\%) with AB and $22(11.2 \%)$ with cystitis. The most frequent etiologies were Escherichia coli, Klebsiella pneumoniae, Enterococcus faecalis, and Pseudomonas aeruginosa. No differences were observed regarding the etiologies, antimicrobial susceptibility patterns, and microbiologic outcomes in $\mathrm{AB}$ vs. cystitis. The treatment of $\mathrm{AB}$ diminished the microbiological cure and increased the rates of microbiologic relapses and reinfections; in addition, treated $\mathrm{AB}$ patients showed a trend of developing symptomatic urinary tract infection in the following six months. The analysis of the data identified the following independent risk factors for cystitis during the six months of follow-up: $A B$ treatment, thymoglobulin induction, previous acute pyelonephritis, and time since transplantation $<1$ year. In summary, considering the lack of clinical benefits of treating $\mathrm{AB}$ and its impact on cystitis development in the follow-up, we support the recommendation of not screening for or treating $\mathrm{AB}$.
\end{abstract}

Keywords: urinary tract infections; kidney recipients; asymptomatic bacteriuria; cystitis; prospective observational cohort

\section{Introduction}

Despite improved surgical techniques, antimicrobial prophylaxis, new immunosuppressive therapies, and better hygiene management of solid organ transplantation recipients (SOTRs), infectious complications remain a major cause of morbimortality in these patients. Urinary tract infections (UTIs) are among the most common infectious complications and the first cause of antibiotics treatment in kidney transplantation recipients (KTRs). The reported incidence of UTIs ranges from $4 \%$ to $75 \%$ in kidney recipients; this wide range could be explained by the heterogeneity of definitions of UTIs, follow-up times, surgical techniques, antimicrobial prophylaxis and immunosuppressive drugs, and design of the studies [1-5]. 
The real effect of UTIs on the outcome of KTRs is not clear. Some studies suggest the absence of association of asymptomatic bacteriuria (AB) and acute cystitis with allograft survival, rejection, renal function, and all-cause short-term mortality. The screening and treatment of $\mathrm{AB}$ did not improve the early outcome after transplantation and increased the risk of suffering multidrug-resistant (MDR) infections in several studies [6,7].

On the other hand, other studies have reported that the burden of UTIs in KTRs is real and high, as suffering from a UTI during the first year after transplantation increases mortality (41\%) and costs per event [8]. Moreover, these patients have a higher risk of MDR infections, which also compromise the outcome [6]. The impact of acute pyelonephritis (APN) on allograft function, although uncertain, determines an adverse outcome when occurring early after transplantation $[4,5,9,10]$.

Despite their clinical frequency, there are unanswered key points regarding the epidemiology of UTIs in KTRs, the differences in resistance patterns depending on the presence of symptoms, and the impact of treatment in the allograft survival, rejection, and mortality. In this study, we aimed to evaluate the epidemiology and clinical manifestation of UTIs and the impact of antimicrobial therapy on $\mathrm{AB}$ in kidney transplantation recipients.

\section{Methods}

\subsection{Study Design}

A prospective observational cohort of consecutive cases of all uncomplicated-cystitis and AB cases in KTRs that attended the outpatient clinic from January 2017 to June 2017, at the Virgen del Rocío University Hospital, Seville, Spain, was analyzed. For the study, only the first episode, in patients with reinfections or relapses, was analyzed. All interventions followed standard clinical practice. The decision to treat the AB and cystitis episodes and the antimicrobial therapy was the choice of the physicians in charge of the patients.

Urine samples were processed within 4-8 h after collection and urine $\mathrm{pH}$ was measured. The Microbiology Service identified the bacterial isolates and performed susceptibility testing by conventional biochemical tests (biochemical testing, pigment production, growth, and colony characteristics). The causative organism and antibiogram were identified using the MicroScan WalkAway ${ }^{\circledR}$ plus System (Beckman Coulter, Switzerland). When the identification was uncertain, it was confirmed by the Bruker Biotyper MALDI-TOF MS system (Bruker Daltonik GmbH, Leipzig, Germany). The European Committee on Antimicrobial Susceptibility Testing (EUCAST) criteria for categorizing susceptibility and resistance patterns were used [11]. Plasma creatinine, and urinary $\mathrm{pH}$, leukocyturia, and nitrites determinations were also performed at inclusion. Demographics, chronic underlying diseases, time from the kidney transplantation, immunosuppressive regimens, clinical data, and antimicrobial therapy were recorded in a standardized database.

Patients were follow-up for six months after inclusion. Urine cultures were performed one and six months after inclusion. Moreover, in patients with urinary symptoms during the follow-up, urine cultures were also performed.

The study was approved by the Ethics Committee of the University Hospitals Virgen del Rocío and Virgen Macarena (2016/186), Seville, Spain.

\subsection{Definitions}

GESITRA/REIPI UTI guidelines [12] were used: Bacteriuria: Urine specimens isolated in quantitative counts $\geq 10^{5}$ colony-forming units (CFU)/mL. Asymptomatic bacteriuria: The presence of bacteriuria in the absence of any symptoms of a UTI. Cystitis: Bacteriuria and clinical manifestations such as dysuria, pollakiuria, urinary urgency, suprapubic pain, and/or hematuria, in the absence of pyelonephritis symptoms. Acute pyelonephritis: The simultaneous presence of bacteriuria and/or bacteremia and fever, with one or more of the following: Lumbar pain (if native kidney involved), renal allograft tenderness (if transplanted kidney involved), chills, or cystitis symptoms. Clinical cure: The resolution of symptoms at seven days after inclusion. Microbiological cure (eradication): Negative urine culture at 7-9 days after the end of treatment. Microbiological reinfection: New episode of 
infection by a different pathogen than the initially isolated. Microbiological relapse: Detection of the same initial pathogen during the month after the inclusion, with a sterile culture before. Microbiological persistence: No negative urine culture in the follow-up. Mortality: Death during the six months prior to the follow-up. Impairment of renal function: Elevation of creatinemia $\geq 0.5 \mathrm{mg} / \mathrm{dl}$.

\subsection{Statistical Analysis}

A descriptive statistical analysis was performed. Continuous variables were expressed as median and interquartile range or mean and standard deviation if adjusted to a normal distribution and evaluated by Shapiro-Wilk or Kolmogorov-Smirnov tests when appropriate. For bivariate analysis, the chi-square test or the Fisher exact test was used for categorical variables; Bonferroni correction was applied when appropriate. For quantitative variables, the Mann-Whitney test or Student's t-test were used based on their distribution. If the variance was not homogeneous (Levene test), an ANOVA test was applied. The relative risks were expressed as odds ratios (ORs) and 95\% confidence intervals (CI). Multivariate models were used to adjust for possible confounding variables. The clinically relevant and statistically significant variables found in the bivariate analysis were included in a matrix analysis (checked by chi-square test for categorical variables and Student's t-test for quantitative variables). Only the independent variables were finally included, which was the multivariate model that described the outcome better. Significance was established at $p<0.05$. All reported $p$-values are based on two-tailed tests. Statistical analyses were performed using SPSS version 18.0 software (SPSS, Chicago, IL, USA).

\section{Results}

\subsection{Characteristics and Outcomes of the Entire Cohort}

Our study included a total of 197 patients, 175 (88.8\%) with AB and $22(11.2 \%)$ with cystitis. Out of the total, $104(52.8 \%)$ were women, and the median age was 59 years (IQR: 48-69). The median time after transplantation was 3.8 years (IQR: 0.8-10). Our study included $58(29.4 \%)$ and $10(6.9 \%)$ patients who received the transplantation in the previous year and month, respectively. The most common immunosuppressive drug combination was mycophenolate (MMF), prednisone, and tacrolimus $(n=124,62.9 \%)$. Induction therapy was performed for 91 (46.1\%) patients: 67 (34.0\%) with basiliximab or daclizumab and $24(12.1 \%)$ with thymoglobulin. Instrumentation of the urinary tract took place in $40(20.3 \%)$ patients, $24(12.2 \%)$ within the previous six months of the UTI diagnosis.

In the previous six months, 80 patients were diagnosed with at least one episode of bacteriuria (40.6\%), 15 with APN (7.6\%), and 10 with cystitis (5.1\%). Patients with cystitis had more frequently detectable urinary nitrites than patients with $\mathrm{AB}(35.7 \% \mathrm{vs} .9 .1 \%$, $p=0.01$, OR 3.4, 95\% CI 1.3-9.1). At inclusion, 70 (35.5\%) patients had viral co-infections: Cytomegalovirus infection in 45 (22.8\%) cases and BK virus infection in 19 (9.6\%). Patients with cystitis were more frequently co-infected with the hepatitis $\mathrm{C}$ virus (HCV) (33.3\% vs. $3.3 \%, p=0.01$, OR $2.3,95 \%$ CI 2.3-18.5). No differences were found in other demographics and transplant-related variables (Table 1).

Most frequent etiologies in all patients were Escherichia coli $(n=89,45.2 \%)$, Klebsiella pneumoniae $(n=30,15.2 \%)$, Enterococcus faecalis $(n=23,11.6 \%)$, and Pseudomonas aeruginosa $(n=13,6.6 \%)$ (Table 1). Etiologies were similar in patients with cystitis and AB. There were $60(30.4 \%)$ isolates resistant to cotrimoxazole, $55(27.9 \%)$ to ciprofloxacin, $38(19.2 \%)$ to amoxicillin-clavulanate, $21(10.6 \%)$ to third- and/or fourth generation cephalosporins, and $19(9.6 \%)$ to fosfomycin. No differences were observed in antimicrobial susceptibility between isolates from patients with cystitis and AB (Table 1). 
Table 1. Baseline, clinical and microbiological features of kidney recipients with bacteriuria.

\begin{tabular}{|c|c|c|c|c|}
\hline Variable & $\begin{array}{c}\text { All Cases } \\
n=197\end{array}$ & $\begin{array}{c}\text { Asymptomatic } \\
\text { Bacteriuria } \\
n=175\end{array}$ & $\begin{array}{c}\text { Cystitis } \\
n=22\end{array}$ & $p$ Value \\
\hline Time from transplant to inclusion (years; median, IQR) & $3.76(0.78-10.3)$ & $3.85(0.77-9.92)$ & $\begin{array}{c}2.35 \\
(0.63-11.4)\end{array}$ & 0.48 \\
\hline Diabetes mellitus- $n(\%)$ & $46(23.4)$ & $42(24.0)$ & $4(18.2)$ & 0.79 \\
\hline Transplant indication- $n(\%)$ & & & & 0.97 \\
\hline Tubulointerstitial & $40(20.3)$ & $35(20.0)$ & $5(22.7)$ & - \\
\hline Glomerulonephritis & $40(20.3)$ & $36(20.6)$ & $4(18.2)$ & - \\
\hline Polycystic kidney disease & $36(18.3)$ & $32(18.3)$ & $4(18.2)$ & - \\
\hline Diabetic nephropathy & $11(5.6)$ & $9(5.1)$ & $2(9.1)$ & - \\
\hline Hypertension/renovascular & $16(8.1)$ & $15(8.6)$ & $1(4.5)$ & - \\
\hline Tumoral & $4(2.0)$ & $4(2.3)$ & $0(0)$ & - \\
\hline Etiology uncertain/unknown & $49(24.9)$ & $43(24.6)$ & $6(27.3)$ & - \\
\hline Charlson index (median, IQR) & $3(2-5)$ & $3(2-5)$ & $4(2-5)$ & - \\
\hline \multicolumn{5}{|l|}{ Induction drug- $n(\%)$} \\
\hline None & $99(50.3)$ & $87(49.7)$ & $12(54.5)$ & - \\
\hline Basiliximab & $56(28.4)$ & $49(28.0)$ & $7(31.8)$ & - \\
\hline Daclizumab & $11(5.6)$ & $10(5.7)$ & $1(4.5)$ & - \\
\hline Thymoglobulin & $24(12.2)$ & $23(13.1)$ & $1(4.5)$ & - \\
\hline \multicolumn{5}{|l|}{ Current immunosuppression- $n(\%)$} \\
\hline MMF & $142(72.1)$ & $2.0)$ & $16(72.7)$ & - \\
\hline Prednisone & $180(91.4)$ & $161(92.0)$ & $19(86.4)$ & - \\
\hline Tacrolimus & $174(88.3)$ & $155(88.6)$ & $19(86.4)$ & - \\
\hline mTOR inhibitors & $10(5.1)$ & $9(5.1)$ & $1(4.5)$ & - \\
\hline Cyclosporine & $12(6.1)$ & $10(5.7)$ & $2(9.1)$ & - \\
\hline Urinary instrumentation- $n(\%)$ & $40(20.3)$ & $35(20.0)$ & $5(22.7)$ & 0.84 \\
\hline Double J stent & $34(17.3)$ & $29(16.6)$ & $5(22.7)$ & - \\
\hline Urethral catheter & $3(1.5)$ & $3(1.7)$ & $0(0)$ & - \\
\hline Nephrostomy & $3(1.5)$ & $3(1.7)$ & $0(0)$ & - \\
\hline Length of instrumentation (days, median, IQR) & $0(0-26)$ & $0(0-26)$ & $0(0-43.5)$ & 0.52 \\
\hline Cotrimoxazole prophylaxis- $n(\%)$ & $32(16.2)$ & $28(16 \%)$ & $4(18.1)$ & - \\
\hline \multicolumn{5}{|l|}{ Etiology- $n(\%)$} \\
\hline Escherichia coli & $89(45.2)$ & $79(45.1)$ & $10(45.5)$ & 0.93 \\
\hline E. coli ESBL-producers & $5(2.5)$ & $5(2.9)$ & $0(0)$ & 0.55 \\
\hline Klebsiella pneumoniae & $30(15.2)$ & $28(16.0)$ & $1(4.5)$ & 0.15 \\
\hline K. pneumoniae ESBL-producers & $5(2.5)$ & $4(2.3)$ & $1(4.5)$ & 0.54 \\
\hline Enterococcus faecalis & $23(11.6)$ & $20(11.4)$ & $3(13.6)$ & 0.76 \\
\hline Pseudomonas aeruginosa & $13(6.6)$ & $11(6.3)$ & $2(9.1)$ & 0.62 \\
\hline Klebsiella oxytoca & $8(4.0)$ & $6(3.4)$ & $2(9.1)$ & 0.27 \\
\hline Proteus mirabilis & $7(3.6)$ & $6(3.4)$ & $1(4.5)$ & 0.75 \\
\hline Morganella morganii & $4(2.0)$ & $4(2.3)$ & $0(0)$ & 0.62 \\
\hline
\end{tabular}


Table 1. Cont.

\begin{tabular}{|c|c|c|c|c|}
\hline Variable & $\begin{array}{c}\text { All Cases } \\
n=197\end{array}$ & $\begin{array}{c}\text { Asymptomatic } \\
\text { Bacteriuria } \\
n=175\end{array}$ & $\begin{array}{c}\text { Cystitis } \\
n=22\end{array}$ & $p$ Value \\
\hline Enterobacter aerogenes & $4(2.0)$ & $3(1.7)$ & $1(4.5)$ & 0.44 \\
\hline Enterobacter cloacae & $3(1.5)$ & $2(1.4)$ & $1(<1)$ & 0.33 \\
\hline Treatment- $n(\%)$ & $75(38.1)$ & $54(30.9)$ & $21(95.5)$ & \\
\hline Ciprofloxacin & $22(11.2)$ & $16(9.1)$ & $6(27.3)$ & 0.01 \\
\hline Fosfomycin & $29(14.7)$ & $19(10.9)$ & $10(45.5)$ & $<0.01$ \\
\hline Amoxicillin-clavulanate & $16(8.1)$ & $12(6.9)$ & 4 (18.2) & 0.07 \\
\hline Cephalosporins & $5(2.5)$ & $4(2.3)$ & $1(4.5)$ & 0.53 \\
\hline Cotrimoxazole & $3(1.5)$ & $3(1.7)$ & $0(0)$ & 0.54 \\
\hline \multicolumn{5}{|l|}{ Antibiotic resistance- $n(\%)$} \\
\hline Ciprofloxacin & $55(27.9)$ & $45(25.7)$ & $10(45.5)$ & 0.16 \\
\hline Fosfomycin & $19(9.6)$ & $17(9.7)$ & $2(9)$ & 0.94 \\
\hline Amoxicillin-clavulanate & $38(19.2)$ & $31(17.7)$ & $7(31.8)$ & 0.12 \\
\hline Cephalosporins & $21(10.6)$ & $16(9.1)$ & $5(22.7)$ & 0.08 \\
\hline Cotrimoxazole & $60(30.4)$ & $53(30.5)$ & $7(31.8)$ & 0.73 \\
\hline
\end{tabular}

MMF: Mycophenolate, mTOR: Mammalian target of rapamycin, ESBL-producers: extended spectrum beta-lactamases-producers. IQR: Interquartile range.

Seventy-five (38.1\%) patients received antimicrobial treatment, with differences in $\mathrm{AB}$ $(n=54,30.8 \%)$ and cystitis $(n=21,95.4 \%)$ cases. The most common antibiotics prescribed were fosfomycin, ciprofloxacin, and amoxicillin-clavulanate, without differences between cystitis and AB (Table 1).

At the one-month follow-up, 191 (96.9\%) out of the 197 patients were cured, and $4(2.0 \%)$ and $2(1.0 \%)$ had cystitis and APN, respectively, without differences between patients with $\mathrm{AB}$ or cystitis at inclusion. At the six-month follow-up, 181 (91.8\%) patients were cured, and $8(4.0 \%)$ and $8(4.0 \%)$ had cystitis and APN, respectively, without differences between patients with $\mathrm{AB}$ or cystitis at inclusion (Table 2). The most frequent etiologies of symptomatic UTIs during the follow-up were E. coli $(64.7 \%)$ and E. faecalis $(29.2 \%)$.

Table 2. Microbiological and clinical outcomes of the total events, asymptomatic bacteriuria and cystitis.

\begin{tabular}{lcccc}
\hline \multicolumn{1}{c}{ Variables } & $\begin{array}{c}\text { Bacteriuria } \\
n=\mathbf{1 9 7}\end{array}$ & $\begin{array}{c}\text { AB } \\
n=\mathbf{1 7 5}\end{array}$ & $\begin{array}{c}\text { Cystitis } \\
\boldsymbol{n = 2 2}\end{array}$ & $p$ Value \\
\hline $\begin{array}{l}\text { One month follow up outcome } \\
\text { Microbiological- } n \text { (\%) }\end{array}$ & & & \\
\hline Cure & $111(56.3)$ & $99(56.7)$ & $12(54.5)$ & 0.51 \\
\hline Persistence & $40(20.3)$ & $35(20.0)$ & $5(22.7)$ & 0.75 \\
\hline Relapse & $11(5.5)$ & $11(6.3)$ & $0(0)$ & 0.26 \\
\hline Re-infection & $14(7.1)$ & $10(5.7)$ & $4(18.2)$ & 0.07 \\
\hline Without follow up data & $21(10.6)$ & $20(11.4)$ & $1(4.5)$ & 0.35 \\
\hline Clinical- $n$ (\%) & & & \\
\hline Asymptomatic & $191(96.9 \%)$ & $170(97.1 \%)$ & $21(95.4 \%)$ & 0.5 \\
\hline Cystitis & $4(2.0)$ & $3(2)$ & $1(4.5)$ & 0.6 \\
\hline APN & $2(1.0)$ & $2(1)$ & $0(0)$ & 0.6 \\
\hline
\end{tabular}


Table 2. Cont.

\begin{tabular}{|c|c|c|c|c|}
\hline Variables & $\begin{array}{c}\text { Bacteriuria } \\
\quad n=197\end{array}$ & $\begin{array}{c}\text { AB } \\
n=175\end{array}$ & $\begin{array}{c}\text { Cystitis } \\
n=22\end{array}$ & $p$ Value \\
\hline \multicolumn{5}{|l|}{$\begin{array}{l}\text { Six months follow up outcome } \\
\text { Microbiological- } n(\%)\end{array}$} \\
\hline Cure & $53(26.9)$ & $48(27.4)$ & $5(22.7)$ & 0.45 \\
\hline Persistence & $34(17.2)$ & $31(17.7)$ & $3(13.6)$ & 0.68 \\
\hline Relapse & $27(13.7)$ & $24(13.7)$ & $3(13.6)$ & 0.96 \\
\hline Re-infection & $37(18.7)$ & $31(17.7)$ & $6(27.2)$ & 0.29 \\
\hline Without follow up data & $58(29.4)$ & $49(28.0)$ & $9(40.9)$ & 0.3 \\
\hline \multicolumn{5}{|l|}{ Clinical- $n(\%)$} \\
\hline Asymptomatic & $181(91.8)$ & $163(93.1 \%)$ & $19(86.3)$ & 0.22 \\
\hline Cystitis & $8(4.0)$ & $6(3.4)$ & $1(4.5)$ & 0.26 \\
\hline $\mathrm{APN}$ & $8(4.0)$ & $6(3.4)$ & $2(9)$ & 0.26 \\
\hline \multicolumn{5}{|l|}{ Graft outcome- $n(\%)$} \\
\hline Graft dysfunction & $10(5.1)$ & $8(4.6)$ & $2(9.1)$ & 0.29 \\
\hline Graft rejection & $4(2.0)$ & $4(2.3)$ & $0(0)$ & 0.17 \\
\hline Graft loss & $1(0.5)$ & $1(0.6)$ & $0(0)$ & 0.17 \\
\hline
\end{tabular}

Regarding the microbiological outcome, at the one-month follow-up, 111 (56.3\%) patients were microbiologically cured. In $40(20.3 \%)$ patients, the bacteriuria persisted, $11(5.5 \%)$ patients relapsed, and $14(7.1 \%)$ patients were re-infected; $21(10.6 \%)$ patients had no urine cultures at this time. At the six-month follow-up, $53(26.9 \%)$ were cured, and $34(17.2 \%), 27(13.7 \%)$, and $37(18.7 \%)$ had persistence, relapse, and re-infection, respectively. No differences were found in microbiological cure at any follow-up time-points regarding $\mathrm{AB}$ or cystitis diagnosis at inclusion (Table 2).

At the six-month follow-up, renal function worsened in 10 (5.1\%) patients, four $(2.0 \%)$ had a graft rejection, and one $(0.5 \%)$ lost the graft. Six (3.0\%) patients died during the six months of follow-up (five AB and one cystitis), none because of the UTI. The graft and survival outcomes of patients with $\mathrm{AB}$ and cystitis were similar (Table 2).

\subsection{Impact of Antibiotic Treatment in AB Outcome}

Among patients with $\mathrm{AB}, 54(30.8 \%)$ received antimicrobial therapy; most common treatments were fosfomycin $(n=19,10.8 \%)$, ciprofloxacin $(n=16,9.1 \%)$, and amoxicillinclavulanate $(n=12,6.8 \%)$. A higher proportion of treated AB patients, when compared to those untreated, received the transplant in the six months before inclusion $(30.2 \%$ vs. $16.7 \%$, $p=0.04$, OR $2.16,95 \%$ CI $1-4.6)$ and had isolates resistant to cotrimoxazole ( $54.1 \%$ vs. $23.2 \%$, $p<0.01$, OR 2.24, 95\% CI 1.4-3.4). Moreover, there were trends to higher creatinine levels before and during the actual episode in vs. untreated $A B$ patients: 1.78 vs. $1.54 \mathrm{mg} / \mathrm{dL}$ $(p=0.07)$ and 1.77 vs. $1.57 \mathrm{mg} / \mathrm{dL}(p=0.08)$, respectively. No differences were found among the rest of the analyzed variables (Table 3 ).

The episodes of symptomatic UTIs in AB patients during the follow-up are summarized in Table 3. After one month, $4(7.4 \%)$ out of 54 treated AB patients presented with a symptomatic UTI (two cystitis and two APN), whereas $1(0.8 \%)$ out of 121 untreated AB patients had a cystitis episode $(p=0.06)$. After six months, $6(11.1 \%)$ treated vs. $4(3.3 \%)$ untreated AB patients had UTI episodes ( $p=0.07$, OR 3.65, 95\% CI 0.98-13.53). 
Table 3. Characteristics of treated and untreated patients with asymptomatic bacteriuria.

\begin{tabular}{|c|c|c|c|c|}
\hline Variables & $\begin{array}{l}\text { Treated AB } \\
\quad n=54\end{array}$ & $\begin{array}{l}\text { Untreated AB } \\
\quad n=121\end{array}$ & OR $(95 \% C I)$ & $p$ Value \\
\hline $\begin{array}{l}\text { Previous creatininemia }(\mathrm{mg} / \mathrm{dL} \text {, } \\
\text { median, IQR) }\end{array}$ & $1.78(0.82-2.75)$ & $1.54(0.86-2.22)$ & $\begin{array}{c}0.08 \\
(0.048-0.212) \\
\end{array}$ & 0.07 \\
\hline $\begin{array}{l}\text { Creatininemia at the time of inclusion } \\
(\mathrm{mg} / \mathrm{dL})\end{array}$ & $1.77(0.98-2.57)$ & $1.57(0.88-2.25)$ & $\begin{array}{c}0.02 \\
(0.126-0.157)\end{array}$ & 0.08 \\
\hline Time since transplant $<6$ months & $16(30.2)$ & $20(16.7)$ & $\begin{array}{c}2.16 \\
(1.013-4.614)\end{array}$ & 0.04 \\
\hline \multicolumn{5}{|l|}{$\begin{array}{l}\text { One month follow up outcome } \\
\text { Microbiological- } n(\%)\end{array}$} \\
\hline Cure & $24(44.4)$ & $75(61.9)$ & $\begin{array}{c}0.49 \\
(0.25-0.94)\end{array}$ & $<0.01$ \\
\hline Persistence & $10(18.5)$ & $25(20.6)$ & $0.9(0.51-1.6)$ & 0.7 \\
\hline Relapse & $7(12.9)$ & $4(3.3)$ & $2.2(1.3-3.69)$ & 0.04 \\
\hline Re-infection & $7(12.9)$ & $3(2.4)$ & $2.4(1.5-3.9)$ & $<0.01$ \\
\hline Without follow up data & $6(11.1)$ & $14(11.6)$ & $0.97(0.48-1.9)$ & 0.95 \\
\hline \multicolumn{5}{|l|}{ Clinical- $n(\%)$} \\
\hline Symptomatic UTI & $4(7.4)$ & $1(0.8)$ & & \\
\hline Cystitis & $2(3.7)$ & $1(0.8)$ & $2.2(0.96-5.1)$ & 0.25 \\
\hline APN & $2(3.7)$ & $2(3.7)$ & $3.3(2.65-4.2)$ & 0.09 \\
\hline \multicolumn{5}{|l|}{$\begin{array}{l}\text { Six months follow up outcome } \\
\text { Microbiological- } n(\%)\end{array}$} \\
\hline Cure & $13(24.1)$ & 37 (30.6) & $0.8(0.4-1.3)$ & 0.3 \\
\hline Persistence & $12(22.2)$ & $25(20.6)$ & $1.06(0.6-1.8)$ & 0.8 \\
\hline Relapse & $12(22.2)$ & $14(11.6)$ & $1.6(1.01-2.7)$ & 0.06 \\
\hline Re-infection & $14(25.9)$ & $23(19)$ & $1.3(0.80-2.12)$ & 0.11 \\
\hline Without follow up data & $13(24.1)$ & $36(29.8)$ & $0.8(0.48-1.38)$ & 0.45 \\
\hline \multicolumn{5}{|l|}{ Clinical- $n(\%)$} \\
\hline Cystitis & $2(3.7)$ & $3(2.5)$ & $1.3(0.44-3.92)$ & 0.66 \\
\hline $\mathrm{APN}$ & $4(7.4)$ & $1(0.8)$ & $2.8(1.8-4.3)$ & 0.03 \\
\hline \multicolumn{5}{|l|}{ Graft outcome- $n(\%)$} \\
\hline Graft rejection & $1(1.8)$ & $3(2.5)$ & $0.8(0.14-4.5)$ & 0.8 \\
\hline Graft dysfunction & $2(3.7)$ & $6(4.9)$ & $0.7(0.14-3.8)$ & 0.7 \\
\hline Graft loss & $0(0.0)$ & $1(0.8)$ & - & 0.7 \\
\hline
\end{tabular}

AB: Asymptomatic bacteriuria, APN: Acute pyelonephritis. IQR: Interquartile range.

A multivariate analysis was performed to evaluate possible confounding variables of the effect of treating $\mathrm{AB}$ at the risk of developing symptomatic UTIs in the six months after inclusion. The following variables were identified as independent risk factors: Use of thymoglobulin as the induction drug $(p<0.01$, OR 8,95\% CI 1.9-34.2), APN after the transplant ( $p<0.01$, OR $12,95 \%$ CI 2.7-53.5), antimicrobial treatment of $\mathrm{AB}(p=0.02$, OR 5, $95 \%$ CI 1.2-20.6), and time since transplantation less than one year ( $p=0.01$, OR 5.7, 95\% CI 1.5-22.2) (Table 4). 
Table 4. Risk factors of symptomatic UTI during the 6 months of follow-up in patients with asymptomatic bacteriuria.

\begin{tabular}{|c|c|c|c|c|c|}
\hline Variables & $\begin{array}{l}\text { Symptomatic UTI } \\
\qquad(n=15)\end{array}$ & $\begin{array}{c}\text { No Symptomatic } \\
\text { UTI } \\
(n=182)\end{array}$ & $\begin{array}{l}\text { Crude OR } \\
(95 \% \text { CI })\end{array}$ & $p$ Value & $\begin{array}{c}\text { Adjusted OR } \\
(95 \% \mathrm{CI})\end{array}$ \\
\hline Urinary pH (median, IQR) & $6.7(6.2-7.2)$ & $6.4(5.9-6.9)$ & $0.11(0.01-0.2)$ & 0.06 & - \\
\hline Previous creatininemia (mg/dl, median, IQR) & $2.07(0.87-3.27)$ & $1.55(0.84-2.27)$ & $0.06(0.01-0.12)$ & 0.02 & - \\
\hline Time after transplant < 1 year (median, IQR) & $9(60)$ & $49(27.22)$ & $4.01(1.4-11.9)$ & 0.01 & $5.7(1.4-22.2)$ \\
\hline Recurrent UTI previous transplant- $n(\%)$ & $4(26.7)$ & $28(15.3)$ & - & 0.3 & - \\
\hline Urinary reflux- $n(\%)$ & $0(0.0)$ & $13(7.1)$ & - & 0.3 & - \\
\hline MMF doses (median, IQR) & $700(435-1045)$ & $750(329-1268)$ & - & 0.6 & - \\
\hline Induction treatment- $n(\%)$ & $11(73.3)$ & $80(43.9)$ & $3.2(1.1-9.7)$ & 0.01 & - \\
\hline No drug & $4(26.7)$ & $95(52.2)$ & $0.36(0.12-1)$ & 0.05 & - \\
\hline Basiliximab & $4(26.7)$ & $52(28.7)$ & - & 1.0 & - \\
\hline Daclizumab & $1(6.6)$ & $10(5.5)$ & - & 1.0 & - \\
\hline Thymoglobulin & $6(40)$ & $18(9.9)$ & $4.6(1.8-11.8)$ & $<0.01$ & $8(1.9-34.2)$ \\
\hline Previous APN post-transplant- $n(\%)$ & $11(73.3)$ & $60(32.9)$ & $4.8(1.6-14.7)$ & $<0.01$ & $12(2.7-53.5)$ \\
\hline Developing UTI 2 months after transplant- $n(\%)$ & $13(86.7)$ & $93(51.1)$ & $4.6(1.1-19.8)$ & 0.03 & - \\
\hline Previous rejection- $n(\%)$ & $2(13.3)$ & $12(6.6)$ & - & 0.49 & - \\
\hline Urinary instrumentation- $n(\%)$ & $5(33.3)$ & $35(19.2)$ & - & 0.36 & - \\
\hline Obstructive uropathy post-transplant- $n(\%)$ & $0(0.0)$ & $10(5.5)$ & - & 0.59 & - \\
\hline Nosocomial acquisition of the AB- $n(\%)$ & $11(73.3)$ & $167(91.7)$ & $0.24(0.06-0.99)$ & 0.04 & - \\
\hline Antibiotic therapy of the AB- $n(\%)$ & $11(73.3)$ & $64(35.2)$ & $4.7(1.5-13.5)$ & 0.02 & $5(1.2-20.6)$ \\
\hline Microbiological cure at 1 month- $n(\%)$ & $4(26.6)$ & $107(58.8)$ & $0.2(0.09-0.854)$ & 0.01 & - \\
\hline
\end{tabular}

UTI: Urinary tract infection, MMF: Mycophenolate, APN: Acute pyelonephritis, AB: Asymptomatic bacteriuria.

Regarding the microbiological outcome, at one-month follow-up, patients with treated $\mathrm{AB}$ experienced a microbiological cure less frequently than those untreated $(44.4 \%$ vs. $61.9 \%, p<0.01$, OR $0.49,95 \%$ CI $0.25-0.94)$. These patients also had a higher number of relapses $(12.9 \%$ vs. $3.3 \%, p<0.05$, OR $2.2,95 \%$ CI $1.3-3.7)$, and re-infections $(12.9 \%$ vs. $2.4 \%$, $p<0.01$, OR 2.4, 95\% CI 1.5-3.9). At the six-month follow-up, microbiological outcomes were similar in treated and untreated $\mathrm{AB}$ (Table 3).

\section{Discussion}

This study shows that antimicrobial resistance is a major issue in kidney recipients with a UTI and that treating $\mathrm{AB}$ in kidney recipients diminish the microbiological cure and increases the rates of microbiologic relapses and reinfections; in addition, treated $A B$ patients showed a trend of developing symptomatic UTIs in the following six months. To our knowledge, this is the largest study to examine the epidemiology and clinical manifestation and impact of antimicrobial therapy on non-complicated UTI in kidney recipients, prospectively.

The most common etiology of non-complicated UTI was E. coli, as described for the general population $[13,14]$; however, the spectrum of etiologies was more diverse than in non-immunocompromised hosts, with a higher frequency of Enterococcus spp. and Pseudomonas aeruginosa infections [1,15]. The high proportion of antimicrobial resistance found must also be highlighted; it is in the range of the proportion described in other studies of kidney recipients (44-77\%) [15-17] and clearly higher than the incidence described in the general population (18-25\%) [14,18].

One-third of the episodes included occurred during the first year after the transplant. It has been described that most episodes of bacteriuria occur early after transplantation [13] Several reasons have been hypothesized to explain these findings, including immunological net status or urinary instrumentation. The close follow-up of early kidney recipients could also have contributed to this finding. 
No differences were found regarding the etiology and antimicrobial susceptibility of bacteriuria according to the presence of symptoms; however, in patients with bacteriuria, the presence of nitrites was associated with urinary symptoms. This finding has not been previously described in kidney recipients; however, a higher sensitivity of urinary nitrites at diagnosing cystitis when screening for bacteriuria in pregnant women, rather than $A B$, has been reported [19].

Hepatitis $C$ virus co-infection also occurred more frequently in patients with cystitis. In the RESITRA cohort, an association is reported between hepatitis $C$ virus serostatus and receiving thymoglobulin or experiencing an upper UTI, which are, at the same time, risk factors for developing a symptomatic UTI in our cohort [20].

Treating $\mathrm{AB}$ did not improve 1-month and 6-month microbiological outcomes. It did not have any impact on the survival of either patients or grafts. This is in accordance with what has been previously described: The persistence of bacteriuria, relapse, or reinfection did not affect the survival, renal function, or allograft function [2,4,10,21-23]. Preventive measures to reduce UTIs in transplant recipients, as antimicrobial prophylaxis, have not been reported to affect either the graft's or patient's survival; however, antimicrobial prophylaxis reduced the incidence of bacteriuria and sepsis in a meta-analysis study [24].

In the present study, treating $\mathrm{AB}$ did not prevent the development of symptomatic UTI in the follow-up, as other studies had already reported $[4,25,26]$. On the contrary, independently treating $\mathrm{AB}$ increased the risk of symptomatic UTIs in the following six months. Some factors were also identified to independently increase the risk of developing a symptomatic UTI during the six months of follow-up: Induction therapy with thymoglobulin, "early" post-transplant period bacteriuria, and previous APN. These factors might be, as previously stated, surrogate markers of the global net-state of immunosuppression and urinary predisposing factors, which might have contributed to a higher risk of symptomatic infection $[2,3,8]$.

The open design of the study is a limitation to be considered. It might explain the increased risk of symptomatic UTI in the follow-up in treated patients with AB. The physician in charge of the patient decided when to treat and, therefore, they more frequently treated patients who had $\mathrm{AB}$, were recently transplanted, had previous episodes of $\mathrm{APN}$, or received thymoglobulin. All these are factors associated with a higher risk of symptomatic UTI in the follow-up, as previously stated. Although treatment of AB was an independent risk factor of developing a symptomatic UTI within six months, the presence of other confounding factors not considered in the present analysis could not be ruled out.

Some randomized trials assessing the impact of treatment $A B$ on kidney recipients have already been reported, with results against treating, within small samples $[4,27]$. Some others are in the process of being concluded or published and might clarify this issue (NCT01871753 and NCT02113774) [27].

\section{Conclusions}

In summary, the high rate of resistant UTIs in kidney recipients and the lack of clinical benefits of treating $\mathrm{AB}$ in the present study support the recommendation of stopping screening and treating $\mathrm{AB}$, while waiting for robust incoming assay results. The risk factors for developing a symptomatic UTI observed in this study might help define a subpopulation that could benefit from specific strategies, such as close follow-up, antimicrobial prophylaxis, or self-antibiotic initiation once symptoms are present.

Author Contributions: S.F. and C.I.-D. complete the data base, analyzed the results and wrote the manuscript. A.S.-B., M.S.-P. and C.G.-C., included the patients in the study and reviewed the manuscript. G.M.-G. worked in the data base. M.E.P.-I. and E.C. conceived the study, designed the experiments, analyzed the results and wrote and revised the manuscript. G.B. investigated. J.P. conceptualized, supervised, wrote and reviewed the manuscript. S.F. and C.I.-D. contributed equally to this work (joint first authors). All authors have read and agreed to the published version of the manuscript. 
Funding: This work was supported by the Instituto de Salud Carlos III, Subdirección General de Evaluación y Fomento de la Investigación, Ministerio de Economía, Industria y Competitividad (PI1701405) and by Plan Nacional de I + D + i 2013-2016 and Instituto de Salud Carlos III, Subdirección General de Redes y Centros de Investigación Cooperativa, Ministerio de Ciencia, Innovación y Universidades, Spanish Network for Research in Infectious Diseases (REIPI RD16/0016/0009)-cofinanced by European Development Regional Fund "A way to achieve Europe", Operative program Intelligent Growth 2014-2020. G.M.-G. was supported by a Río Hortega research contract from the Instituto de Salud Carlos III.). M.E.P.I. is supported by the "Contract to Access to the Spanish System of Research and Innovation, V Research Program of the University of Seville" (USE13901-D) grant.

Conflicts of Interest: None of the authors has a conflict of interest to declare.

\section{References}

1. Vidal, E.; Torre-Cisneros, J.; Blanes, M.; Montejo, M.; Cervera, C.; Aguado, J.; Len, O.; Carratalá, J.; Cordero, E.; Bou, G.; et al. Bacterial urinary tract infection after solid organ transplantation in the RESITRA cohort. Transpl. Infect. Dis. 2012, 14, 595-603. [CrossRef] [PubMed]

2. Pellé, G.; Vimont, S.; Levy, P.P.; Hertig, A.; Ouali, N.; Chassin, C.; Arlet, G.; Rondeau, E.; Vandewalle, A. Acute Pyelonephritis Represents a Risk Factor Impairing Long-Term Kidney Graft Function. Arab. Archaeol. Epigr. 2007, 7, 899-907. [CrossRef] [PubMed]

3. Alangaden, G.J.; Thyagarajan, R.; Gruber, S.A.; Morawski, K.; Garnick, J.; El-Amm, J.M.; West, M.S.; Sillix, D.H.; Chandrasekar, P.H.; Haririan, A. Infectious complications after kidney transplantation: Current epidemiology and associated risk factors. Clin. Transplant. 2006, 20, 401-409. [CrossRef]

4. Origüen, J.; López-Medrano, F.; Fernández-Ruiz, M.; Polanco, N.; Gutiérrez, E.; González, E.; Mérida, E.; Ruiz-Merlo, T.; MoralesCartagena, A.; Asín, M.A.P.; et al. Should Asymptomatic Bacteriuria Be Systematically Treated in Kidney Transplant Recipients? Results from a Randomized Controlled Trial. Arab. Archaeol. Epigr. 2016, 16, 2943-2953. [CrossRef]

5. Singh, R.; Bemelman, F.J.; Geerlings, S.E. Asymptomatic bacteriuria in renal allograft recipients: Not so innocent after all? Futur. Microbiol. 2016, 11, 1-3. [CrossRef]

6. Linares, L.; Cervera, C.; Cofán, F.; Ricart, M.; Esforzado, N.; Torregrosa, V.; Oppenheimer, F.; Campistol, J.; Marco, F.; Moreno, A. Epidemiology and Outcomes of Multiple Antibiotic-Resistant Bacterial Infection in Renal Transplantation. Transplant. Proc. 2007, 39, 2222-2224. [CrossRef]

7. Fontserè, S.; Chacón-Mora, N.; Cordero, E. Review of Bacterial Urinary Tract Infection in Kidney Transplant Recipients: Incidence, Risk Factors and Impact on the Graft Survival. Int. J. Transplant. Res. Med. 2017, 3, 1-4. [CrossRef]

8. Naik, A.S.; Dharnidharka, V.R.; Schnitzler, M.A.; Brennan, D.C.; Segev, D.L.; Axelrod, D.; Xiao, H.; Kucirka, L.; Chen, J.; Lentine, K.L. Clinical and economic consequences of first-year urinary tract infections, sepsis, and pneumonia in contemporary kidney transplantation practice. Transpl. Int. 2016, 29, 241-252. [CrossRef] [PubMed]

9. Singh, R.; Geerlings, S.E.; Bemelman, F.J. Asymptomatic bacteriuria and urinary tract infections among renal allograft recipients. Curr. Opin. Infect. Dis. 2015, 28, 112-116. [CrossRef]

10. Fiorante, S.; Fernández-Ruiz, M.; López-Medrano, F.; Lizasoain, M.; Lalueza, A.; Morales, J.M.; San-Juan, R.; Andrés, A.; Otero, J.R.; Aguado, J.M. Acute graft pyelonephritis in renal transplant recipients: Incidence, risk factors and long-term outcome. Nephrol. Dial. Transplant. 2010, 26, 1065-1073. [CrossRef]

11. The European Committee on Antimicrobial Susceptibility Testing. Breakpoint tables for interpretation of MICs and zone diameters. Version 11.0. 2021. Available online: https://www.eucast.org/fileadmin/src/media/PDFs/EUCAST_files/Breakpoint_tables/ v_11.0_Breakpoint_Tables.pdf (accessed on 1 January 2021).

12. Vidal, E.; Cervera, C.; Cordero, E.; Armiñanzas, C.; Carratalá, J.; Cisneros, J.M.; Fariñas, M.C.; López-Medrano, F.; Moreno, A.; Muñoz, P.; et al. Management of urinary tract infection in solid organ transplant recipients: Consensus statement of the Group for the Study of Infection in Transplant Recipients (GESITRA) of the Spanish Society of Infectious Diseases and Clinical Microbiology (SEIMC) and the Spanish Network for Research in Infectious Diseases (REIPI). Enferm. Infecc. y Microbiol. Clin. 2015, 33, 679.e1-679.e21.

13. Fiorentino, M.; Pesce, F.; Schena, A.; Simone, S.; Castellano, G.; Gesualdo, L. Updates on urinary tract infections in kidney transplantation. J. Nephrol. 2019, 32, 751-761. [CrossRef] [PubMed]

14. Butler, C.C.; Francis, N.; Thomas-Jones, E.; Llor, C.; Bongard, E.; Moore, M.; Little, P.; Bates, J.; Lau, M.; Pickles, T.; et al. Variations in presentation, management, and patient outcomes of urinary tract infection: A prospective four-country primary care observational cohort study. Br. J. Gen. Pract. 2017, 67, e830-e841. [CrossRef]

15. Khosravi, A.D.; Montazeri, E.A.; Ghorbani, A.; Parhizgari, N. Bacterial urinary tract infection in renal transplant recipients and their antibiotic resistance pattern: A four-year study. Iran J. Microbiol. 2014, 6, 74-78. [PubMed]

16. Origüen, J.; Fernández-Ruiz, M.; López-Medrano, F.; Ruiz-Merlo, T.; González, E.; Morales, J.; Fiorante, S.; San-Juan, R.; Villa, J.; Orellana, M.; et al. Progressive increase of resistance in Enterobacteriaceae urinary isolates from kidney transplant recipients over the past decade: Narrowing of the therapeutic options. Transpl. Infect. Dis. 2016, 18, 575-584. [CrossRef] 
17. Azap, Ö.; Togan, T.; Yeşilkaya, A.; Arslan, H.; Haberal, M. Antimicrobial Susceptibilities of Uropathogen Escherichia coli in Renal Transplant Recipients: Dramatic Increase in Ciprofloxacin Resistance. Transplant. Proc. 2013, 45, 956-957. [CrossRef]

18. Rossignol, L.; Vaux, S.; Maugat, S.; Blake, A.; Barlier, R.; Heym, B.; Le Strat, Y.; Blanchon, T.; Hanslik, T.; Coignard, B. Incidence of urinary tract infections and antibiotic resistance in the outpatient setting: A cross-sectional study. Infection 2016, 45, 33-40. [CrossRef]

19. Demilie, T.; Beyene, G.; Melaku, S.; Tsegaye, W. Diagnostic accuracy of rapid urine dipstick test to predict urinary tract infection among pregnant women in Felege Hiwot Referral Hospital, Bahir Dar, North West Ethiopia. BMC Res. Notes 2014, 7, 481. [CrossRef] [PubMed]

20. López-Medrano, F.; Fernández-Ruiz, M.; Morales, J.M.; San-Juan, R.; Cervera, C.; Carratalá, J.; Torre-Cisneros, J.; Gavaldá, J.; Muñoz, P.; Len, Ó.; et al. Impact of Hepatitis C Virus Infection on the Risk of Infectious Complications After Kidney Transplantation: Data From the RESITRA/REIPI Cohort. Transplantation 2011, 92, 543-549. [CrossRef]

21. Moradi, M.; Abbasi, M.; Moradi, A.; Boskabadi, A.; Jalali, A. Effect of antibiotic therapy on asymptomatic bacteriuria in kidney transplant recipients. Urol. J. 2005, 2, 32-35. [PubMed]

22. Ariza-Heredia, E.J.; Beam, E.N.; Lesnick, T.G.; Cosio, F.G.; Kremers, W.K.; Razonable, R.R. Impact of urinary tract infection on allograft function after kidney transplantation. Clin. Transplant. 2014, 28, 683-690. [CrossRef]

23. Capocasale, E.; De Vecchi, E.; Mazzoni, M.; Valle, R.D.; Pellegrino, C.; Ferretti, S.; Sianesi, M.; Iaria, M. Surgical Site and Early Urinary Tract Infections in 1000 Kidney Transplants With Antimicrobial Perioperative Prophylaxis. Transplant. Proc. 2014, 46, 3455-3458. [CrossRef]

24. Green, H.; Rahamimov, R.; Gafter, U.; Leibovitci, L.; Paul, M. Antibiotic prophylaxis for urinary tract infections in renal transplant recipients: A systematic review and meta-analysis. Transpl. Infect. Dis. 2011, 13, 441-447. [CrossRef] [PubMed]

25. Green, H.; Rahamimov, R.; Goldberg, E.; Leibovici, L.; Gafter, U.; Bishara, J.; Mor, E.; Paul, M. Consequences of treated versus untreated asymptomatic bacteriuria in the first year following kidney transplantation: Retrospective observational study. Eur. J. Clin. Microbiol. Infect. Dis. 2012, 32, 127-131. [CrossRef]

26. Sabé, N.; Oriol, I.; Melilli, E.; Manonelles, A.; Bestard, O.; Polo, C.; Arcos, I.L.; Perelló, M.; Garcia, D.; Riera, L.; et al. Antibiotic Treatment Versus No Treatment for Asymptomatic Bacteriuria in Kidney Transplant Recipients: A Multicenter Randomized Trial. Open Forum Infect. Dis. 2019, 6, ofz243. [CrossRef] [PubMed]

27. Rosado-Canto, R.; Carrillo-Pérez, D.L.; Arreola-Guerra, J.M.; Sifuentes-Osornio, J. Asymptomatic bacteriuria in kidney transplant recipients: The challenge in the first 8 weeks. Transpl. Infect. Dis. 2018, 20, e12895. [CrossRef] [PubMed] 